\title{
A multisample dissolution dynamic nuclear polarization system for serial injections in small animals
}

\section{Journal Article}

\section{Author(s):}

Krajewski, Marcin; Wespi, Patrick; Busch, Julia; Wissmann, Lukas; Kwiatkowski, Grzegorz; Steinhauser, Jonas; Batel, Michael; Ernst, Matthias (D); Kozerke, Sebastian

Publication date:

2017-02

Permanent link:

https://doi.org/10.3929/ethz-b-000114070

Rights / license:

In Copyright - Non-Commercial Use Permitted

Originally published in:

Magnetic Resonance in Medicine 77(2), https://doi.org/10.1002/mrm.26147 


\section{A Multi-Sample Dissolution DNP System for Serial Injections in Small Animals}

Marcin Krajewski ${ }^{1,2}$, Patrick Wespi ${ }^{1}$, Julia Busch ${ }^{1}$, Lukas Wissmann ${ }^{1}$, Grzegorz Kwiatkowski ${ }^{1}$, Jonas Steinhauser ${ }^{1}$, Michael Batel ${ }^{2}$, Matthias Ernst ${ }^{2}$, Sebastian Kozerke ${ }^{1}$

${ }^{1}$ Institute for Biomedical Engineering, University and ETH Zurich, Switzerland;

${ }^{2}$ Laboratory of Physical Chemistry, ETH Zurich, Switzerland

Running title: $\quad$ Multi-sample dissolution DNP polarizer for small animal research

Submission: $\quad$ MRM Technical Note

Word Count: $\quad 2866$

Mailing Address: $\quad$ Sebastian Kozerke, PhD

Institute for Biomedical Engineering

University and ETH Zurich

Gloriastrasse 35

8092 Zurich

Tel.: + + 41446323549

FAX: $\quad+41446321193$

Email: kozerke@biomed.ee.ethz.ch 


\section{Abstract}

\section{Purpose}

A number of experimental in-vivo applications of dissolution Dynamic Nuclear Polarization (DNP) requires rapid successive injections of hyperpolarized substrates. Here we present the design and performance of a home-built multi-sample dissolution DNP setup for small animal research.

\section{Methods}

The DNP setup consists of a commercial wide-bore magnet charged to $3.35 \mathrm{~T}$, a cryostat, a $94 \mathrm{GHz}$ microwave source and a custom-built skeleton, which accommodates four identical sample sticks. Each sample stick features a dissolver locked into the skeleton port and a lifter, which permits moving the sample cup out of the liquid helium bath for dissolution.

\section{Results}

The dissolution of the first sample was triggered after 2 hours of polarization build up during single-shot operation of the cryostat. Thereafter a time window of 75-90 min was available to dissolve the remaining three polarized samples. The average liquid state-polarization over all four sticks was measured as $18.7 \pm 2.3 \%$ for [1-13C] pyruvate at $30 \mathrm{sec}$ after dissolution. In-vivo applicability of the setup using serial injections of [1-13C] pyruvate to study cardiac metabolism in rats revealed good reproducibility.

\section{Conclusion}

The simple four-sample DNP insert described here provides reproducible liquid state polarization of [1-13C] pyruvate and allows for rapid repeat injections in small animals.

\section{Key words}

Dynamic nuclear polarization, dissolution DNP, multi-sample DNP, metabolic imaging, small animal 


\section{Introduction}

Dissolution Dynamic Nuclear Polarization (DNP) (1) has gained considerable attention as it provides $>10^{\prime} 000$-fold signal enhancement of relevant molecules for MR imaging and spectroscopy studies. In DNP, highly polarized electron spins are used to increase the polarization of nuclei of interest in a low-temperature environment using microwave irradiation (2). The transfer of polarization requires between $30 \mathrm{~min}$ and 3 hours depending on various parameters such as the static magnetic field strength, the radicals used or the type of nuclei involved. After polarizing the nuclei, samples are rapidly dissolved using pressurized, heated water and the final solution is then available for in-vivo studies (1). With reasonably sized molecules, $T_{1}$ relaxation times in liquid state are sufficiently long to enable MR imaging of the turnover of metabolically active substrates (3-7). To separate different metabolic products from the injected substrate, chemical-shift selective imaging or decoding is exploited providing unparalleled insights into in-vivo metabolism in real time (8-11). The method is promising for both oncology (12) and cardiovascular applications (13). Recent work has also demonstrated the feasibility and safety of the concept in humans provided that sterile compounding is possible (14).

A key strength of dissolution DNP using endogenous substrates is the repeatability of injections thereby providing access to metabolic information at various time points during disease progression and therapy. To study transient metabolic changes during pharmacological or structural interventions, however, consecutive injections in rapid succession are required. For example, shortage of oxygen supply to the heart leads to insufficient production of high-energy phosphates and impairment of contractile function within minutes (15). 
Different single-sample DNP polarizers have been described in the literature $(1,16-19)$ following similar concepts. The original singe-sample, integrated cryostat design by Ardenkjaer-Larsen et al. (1) has been further developed and commercialized (HyperSense, Oxford Instruments, UK). While the combined magnet/cryo design of the HyperSense device has advantages, it also complicates maintenance. To this end, home-built polarizers have primarily been constructed using standard vertical-bore NMR magnets with field strengths between 3.35 and 7 Tesla and with separate cryostats (16-22). Dedicated home-built inserts are used to implement the DNP functionality including capabilities to assess NMR and ESR signals for diagnostic purposes.

In recent work the potential of cross-polarization to shorten the long polarization build-up times in dissolution DNP has been highlighted $(23,24)$. In addition, application of an additional modulation of the microwave irradiation field proved to reduce the build-up time of ${ }^{1} \mathrm{H}$ nuclei in experiments conducted with nitroxide radicals (25). Nevertheless, sample loading and polarization times remain relatively long compared to requirements of many interventional in-vivo studies. Given the long time constants of polarization build-up and hence the inability to provide hyperpolarized samples for rapid repeat metabolic studies, devices for concurrent polarization of multiple samples have been proposed $(20,21,26)$. While the commercial foursample sterile device by Ardenkjaer-Larsen et al. (26) is designed to provide large sample volumes for potential human applications, its purchasing and running costs may not be justified for small-animal imaging experiments.

The objective of the present work is to describe the design and performance of a low-cost four-sample dissolution DNP insert for standard wide-bore NMR magnets and cryostats providing hyperpolarized liquid-state samples with a minimum latency of $3 \mathrm{~min}$. The 
applicability of the device is demonstrated using sequential dissolutions and imaging of cardiac metabolism in rats at four different time points within one hour.

\section{Methods}

Beside the DNP insert, the general setup presented here is similar to our previous multisample polarizer (20). In brief, a standard NMR cryostat (Oxford Instruments, UK) is mounted in a shielded $7 \mathrm{~T}$ wide-bore $(89 \mathrm{~mm}$ ) magnet (Bruker Biospin, Switzerland) charged to $3.35 \mathrm{~T}$. The DNP insert operates with a $94 \mathrm{GHz}$ microwave source (Elva, Russia). A generic data acquisition system with multi-purpose interface (National Instruments, USA) is used in conjunction with home-built LabView software (National Instruments, USA) to control the system.

\section{Cryogenic system}

The cryogenic system includes a variable-temperature insert, a liquid-helium transfer line, and vacuum pumps. Large-diameter tubing (ISO-KF 40) is used to minimize the pressure drop along the exhaust line. A roots-pump (Okta 500, Pfeiffer Vacuum, Switzerland) in series with a dual-stage rotary-vane pump (Duo65, Pfeiffer Vacuum, Switzerland) is employed to pump on the helium. To fill the system, liquid helium is drawn through the transfer line from a liquidhelium supply dewar. A needle valve is used to control the liquid helium flow into the bottom of the cryostat and the sample space. A helium recovery system is connected to recycle helium gas and to reduce the overall running costs.

To prepare the system for operation, the cryostat is pumped with the exhaust gas guided through a tube surrounding the helium-supply capillary within the transfer line, thereby precooling helium. To achieve temperatures below $3 \mathrm{~K}$, the cryostat is operated in single-shot 
mode after filling. In this case helium is pumped directly through an exhaust port on the cryostat. The liquid-helium level is monitored using a capacitive sensor built from two stainless steel tubes. Temperature sensors (Cernox, USA) are mounted in the cryostat vacuum shield at the bottom of the helium bath and on the copper baffle above the sample space.

\section{DNP insert}

A custom-built skeleton, which can accommodate up to four identical sample sticks for concurrent polarization, is inserted into the cryostat (Figure 1). The maximum sample volume per stick is $135 \mu \mathrm{l}$. Each sample stick features a dissolver locked into the skeleton port upon loading and a lifter which permits moving the sample cup by approximately $5 \mathrm{~cm}$ longitudinally for polarization/dissolution (Figure 2). For polarization the sample is in the lower position within the liquid helium bath and lifted above the liquid-helium level for dissolution. The lifter is operated using a mechanical pulling rod. Upon pulling the lifter, the sample holder docks into the dissolver forming a chamber separated from the cryostat vacuum.

\section{Microwave system}

Microwaves are guided from the source by a short piece of WR10 waveguide coupled to a circulator followed by a tapered transition to an oversized WR28 waveguide. In the skeleton the waveguide is fed centrally between the sample sticks to the magnet center using an Ebend and a transition from copper to stainless steel including a mylar window as a vacuum seal. The internal flanges are custom-made due to space limitations. No dedicated microwave cavity or horn antenna is used. To confine the microwave energy to some extent in the sample space, the cryostat walls and lowest skeleton baffles are made from copper. 


\section{NMR diagnostics}

For NMR signal monitoring a single loop " $\mathrm{B}$ " shaped coil was mounted between the sticks in the symmetry plane of the waveguide and connected to a $3.5 \mathrm{~mm}, 50 \mathrm{Ohm}$ semi-rigid transmission line consisting of stainless steel and copper (upper part) / beryllium copper (lower part) to reduce thermal conductivity into the cryo space.

\section{Operation}

An experiment cycle starts with sample-stick loading at $10-20 \mathrm{~K}$ cryostat temperature, filling and cooling the cryostat with liquid helium. Thereafter, the helium inlet is closed and the pumps are operated at maximum power while directly connected to the cryostat. Using microwave irradiation, all four samples are polarized simultaneously. The sample sticks are lifted and dissolved independently from each other without pressurizing the cryostat. For dissolution a selected stick is connected to the dissolution device, the water heater is filled with dissolution buffer (Tris), pressurized and heated to $170^{\circ} \mathrm{C}$. Subsequently, the sample is moved up by $44 \mathrm{~mm}$ to pull it above the liquid-helium level (Figure 2) and the dissolution is triggered. During this process microwaves are temporarily switched off. The valve on the collector opens automatically and the sample fluid is collected into a syringe. At the end of an experiment cycle the liquid-helium flow is restarted, the cryostat pressurized to retract the empty sample sticks and a new cycle can be started.

\section{In-vivo measurements}


Four healthy female Sprague Dawley rats (250-300g) were anaesthetized using 1-2\% isoflurane in an air/oxygen (4:1) mixture, endotracheally intubated and placed in a Bruker Biospec $9.4 \mathrm{~T}$ small animal MR system. Body temperature was kept at $37-38{ }^{\circ} \mathrm{C}$. The animals were mechanically ventilated at $60 \mathrm{bpm}$ and heart rate was continuously monitored (300-400 bpm). To enhance bicarbonate signal, the animals received an iv glucose infusion (Bichsel AG, Interlaken, Switzerland; $15 \mathrm{mg}$ glucose per $\mathrm{kg}$ of body weight per minute). All animal experiments were performed in adherence to the Swiss law of Animal Protection and approved by the Zurich cantonal veterinary office. A birdcage dual ${ }^{1} \mathrm{H} /{ }^{13} \mathrm{C}$ coil (Rapid Biomedical, Wurzburg, Germany) was used for transmission, and a ${ }^{13} \mathrm{C}$ surface coil from the same vendor was used for signal reception. Linear order shimming was performed by minimizing the water line width using a localized spectroscopic pulse sequence with a voxel covering the left ventricle. The shimming sequence was triggered to respiratory and cardiac motion. Samples containing $50.8 \mu \mathrm{l}$ of [1-13C] pyruvic acid, $13.5 \mathrm{mM}$ trityl radical and $1 \mathrm{mM}$ Gadolinium (Dotarem, Guerbet, France) were polarized and dissolved using $8 \mathrm{ml}$ of $50 \mathrm{mM}$ Tris/ $\mathrm{NaCl}$ buffer $(\mathrm{pH} 7.5)$. The liquid was collected in a collector containing $650 \mu \mathrm{l}$ of $1 \mathrm{M}$ $\mathrm{NaOH}$. Rats were injected $1 \mathrm{ml}$ of the final solution of $44 \mathrm{mM}$ [1-13C] pyruvate. A spatialspectral excitation pulse was used in combination with a multi-echo EPI readout (number of echoes 7, echo time $5.04 \mathrm{~ms}$, echo time increment $0.383 \mathrm{~ms}$, FOV $60 \times 40 \mathrm{~mm}^{2}$, matrix $48 \times 22$, partial Fourier factor 0.69 , in-plane resolution $1.25 \times 1.25 \mathrm{~mm}^{2}$, slice thickness $5 \mathrm{~mm}$ ) to dynamically image the conversion of pyruvate into lactate and bicarbonate with a repetition interval of $1.5 \mathrm{~s}$ during a total scan duration of $5 \mathrm{~min}$ (27). Metabolic images were reconstructed using the IDEAL approach (28) resolving pyruvate, lactate, bicarbonate, pyruvate hydrate and alanine resonances. Using the spatial-spectral excitation pulse, the nominal flip angles on the pyruvate, lactate and bicarbonate resonances were 4, 19 and 19 
deg, respectively. Four repeat injections were performed in each animal at 0, 20, 40 and 60 min to demonstrate rapid sequential dissolutions. Signal intensity time curves of lactate and bicarbonate were integrated to obtain the area-under-the-curve (AUC). Lactate-tobicarbonate AUC ratios were calculated in four myocardial segments to assess in-vivo reproducibility. 


\section{Results}

\section{System performance}

Optimum microwave output power at the source was $40 \mathrm{~mW}$. The temperature near the sample was $\leq 1.35 \mathrm{~K}$ during single-shot mode. Upon dissolution of an individual sample, the temperature increased to about $2 \mathrm{~K}$ but returned back to the initial value within $2-10 \mathrm{~min}$ depending on the remaining helium level (Figure 3). The helium consumption during singleshot operation was approximately $115 \mathrm{ml} / \mathrm{h}$. The heat load introduced by the dissolution of the four sticks consumed an additional amount of $180 \mathrm{ml}$ of helium. Given the helium level prior to the dissolution of the first sample, a time window of $75-90$ min was available to dissolve the remaining three samples. The black line of the NMR signal build-up curve shown in Figure 3 is corrected for differences in coil loading and sample volume associated with successive dissolution/removal of samples. Liquid state-polarization for the four sticks was measured from a total of 24 experiments as $18.7 \pm 2.3 \%$ at $t=30 \mathrm{sec}$ after dissolution and extrapolated back to $t=0 \mathrm{sec}$ resulting in polarization values of $36.4 \pm 4.5 \%$ for measured $\mathrm{T}_{1}$ values of $45.2 \pm 4.0 \mathrm{sec}$ at $9.4 \mathrm{~T}$. Individual polarization levels of dissolved [1-13C] pyruvate for sticks \#1-4 are given in Figure 4. The order of dissolution of stick \#1-4 was randomized during 5-7 runs per stick.

The total time required for one experiment cycle includes sample loading ( $5 \mathrm{~min}$ ), pre-cooling (15 min), filling of the cryostat (40 min), switch to single-shot mode (10 min), DNP polarization buildup (90-120 min), sample dissolutions, stick retrieval and reestablishing of helium transfer (5-10 min). Accordingly, the first sample is available for dissolution after $160 \mathrm{~min}$. The dissolution of the last sample should happen within 90 min after the first dissolution given the helium consumption in single-shot mode. The minimum interval between successive dissolutions is currently $3 \mathrm{~min}$, which is given by refilling, switching and connecting the single 
water heater to different sticks. A volume of $8 \mathrm{ml}$ of the dissolution medium was found to give reproducible dissolution results.

\section{In-vivo measurements}

Example metabolic maps of pyruvate, lactate and bicarbonate of the heart and corresponding signal intensity time curves obtained sequentially in one animal are shown in Figure 5 . In the left-ventricular blood pool, peak pyruvate SNR across all four animals was measured to be 41.9 \pm 26.7 (mean \pm SD), while lactate and bicarbonate SNR in the myocardial segments were $9.9 \pm 3.3$ and $8.9 \pm 2.5$, respectively. The lactate-to-bicarbonate AUC ratio over all animals and dissolutions was $1.22 \pm 0.23$. The difference in lactate-to-bicarbonate AUC ratio between injections was $2.3 \pm 15.7 \%, 0.3 \pm 16.8,0.7 \pm 8.8 \%$ and $0.7 \pm 13.2 \%$ for anterior, septal, inferior and lateral myocardial segments, respectively. Intra-subject reproducibility of the lactate-tobicarbonate ratio was assessed using the relative mean absolute deviation resulting in $6.1 \%$, $8.8 \%, 3.0 \%$ and $10.8 \%$ for the four individual animals, respectively. There was no trend seen in the lactate-to-bicarbonate ratio as a function of injection across this small population. 


\section{Discussion}

A home-built, multi-sample dissolution DNP insert has been presented permitting dissolution of up to four hyperpolarized samples within a $75-90$ min time window with a minimum interval of 3 min between consecutive dissolutions. In-vivo, sequential dissolution and metabolic imaging of pyruvate metabolism in the rat heart with a time interval of 20 min has been demonstrated with on overall good intra-subject reproducibility of lactate-tobicarbonate ratios in four myocardial segments across four animals. The interval of $20 \mathrm{~min}$ inbetween injections was chosen to reduce stress to the animals.

Operation of the dissolution DNP system is facilitated by the fact that all components remain in the cryostat under vacuum and switching of microwave and dissolution paths as required with other systems is not necessary $(1,16-19,21)$. Compared to other multi-sample system published elsewhere (29) the dissolution volume could be restricted to $8 \mathrm{ml}$ hence minimizing dead volumes during prospective in-vivo experiments in small animals. Further volume reduction to $6 \mathrm{ml}$ was tested and is possible. Alternatively, inert fluids with subsequent separation could be considered (30) to reduce the amount of substrate.

Liquid-state polarization levels and $T_{1}$ time constants of [1-13C] pyruvate obtained in this work are in line with literature values. To increase polarization levels beyond those obtained here, the system can straightforwardly be ramped to twice the field $(6.7 \mathrm{~T})$. Given the low microwave power required at $3.35 \mathrm{~T}(40 \mathrm{~mW})$, a microwave frequency multiplier can be used in conjunction with the current source, which provides a maximum power of $200 \mathrm{~mW}$. In addition, further improvement in liquid state polarization available at the time of injection could be obtained by reducing the sample transfer time with a dedicated shuttling system including magnetic shelter tunnel and/or automatic injection setup (31,32).Liquid-state polarization levels and $T_{1}$ time constants of [1-13C] pyruvate obtained in this work are in line 
with literature values. To increase polarization levels beyond those obtained here, the system can straightforwardly be ramped to twice the field $(6.7 \mathrm{~T})$. Given the low microwave power required at 3.35 $\mathrm{T}(40 \mathrm{~mW})$, a microwave frequency multiplier can be used in conjunction with the current source, which provide a maximum power of $200 \mathrm{~mW}$. To reduce polarization times when working with nitroxide radicals, microwave frequency modulation (25) may readily be employed as the transmission line of the system is sufficiently broadband. The application of cross-polarization $(23,24)$ is currently limited by the inhomogeneous B1+field and the quality factor of the NMR coil in the sample space. To improve B1+ homogeneity either individual coils for each sample need to be fitted or a large volume-resonator inside the cavity should be used. These options, however, remain to be evaluated in detail. In addition, further improvement in liquid state polarization available at the time of injection could be obtained by reducing the sample transfer time with a dedicated shuttling system including magnetic shelter tunnel and/or automatic injection setup $(31,32)$.

The solid-state NMR signal reported in Figure 3 was corrected for intrinsic signal contributions from the sample holder and lifter material as well as for differences in coil loading and sample volume depending on different combinations of sample stick states (polarization/dissolution). To address any inaccuracies in this correction step, separate NMR coils for each stick need to be accommodated.

The duration of one experiment cycle is currently limited by the helium consumption in singleshot mode. To address this limitation an additional helium reservoir can be inserted into the cryostat to allow for rapid helium refilling or continuous flow operation (19). Our current insert geometry was designed with this aspect in mind and can accommodate an additional helium reservoir in the future. 
The material cost, besides the magnet price, of the presented system is approximately $80^{\prime} 000$ US\$ and includes the cost for the vacuum pumps, microwave source, NMR console, cryostat, transfer line, DNP insert components and NMR diagnostics. The cost of the NMR magnet adds another 100 '000 US\$. To keep the cost of operation low, the system was connected to our institutional helium recovery system.

\section{Conclusion}

In this work a simple four-sample DNP insert suitable for standard NMR magnets and heliumtemperature cryostat components was constructed and demonstrated to provide reproducible liquid state polarization of [1-13C] pyruvate for rapid repeat injections in small animals. 


\section{Acknowledgements}

Stephen Wheeler is thanked for manufacturing the DNP insert components; Andreas Hunkeler is acknowledged for expert advice. Experimental assistance and support by Georgios Batsios is gratefully acknowledged. The authors accredit support from the Swiss National Science Foundation, grant \#CR3213_132671/1 and the Commission for Technology and Innovation CTI, grant 14727.1 PFLS-LS. 


\section{References}

1. Ardenkjaer-Larsen JH. Increase in signal-to-noise ratio of $>10,000$ times in liquid-state NMR. Proceedings of the National Academy of Sciences 2003;100:10158-10163. doi: 10.1073/pnas.1733835100.

2. Abragam A, Goldman M. Principles of dynamic nuclear polarisation. Rep. Prog. Phys. 1978;41:395-467. doi: 10.1088/0034-4885/41/3/002.

3. Golman K. Molecular imaging with endogenous substances. Proceedings of the National Academy of Sciences 2003;100:10435-10439. doi: 10.1073/pnas.1733836100.

4. Golman K. Real-time metabolic imaging. Proceedings of the National Academy of Sciences 2006;103:11270-11275. doi: 10.1073/pnas.0601319103.

5. Merritt ME, Harrison C, Storey C, Jeffrey FM, Sherry AD, Malloy CR. Hyperpolarized $13 C$ allows a direct measure of flux through a single enzyme-catalyzed step by NMR. Proceedings of the National Academy of Sciences 2007;104:19773-19777. doi:

10.1073/pnas.0706235104.

6. Gallagher FA, Kettunen MI, Day SE, et al. Magnetic resonance imaging of $\mathrm{pH}$ in vivo using hyperpolarized 13C-labelled bicarbonate. Nature 2008;453:940-943. doi:

10.1038/nature07017.

7. Brindle KM, Bohndiek SE, Gallagher FA, Kettunen MI. Tumor Imaging Using Hyperpolarized C-13 Magnetic Resonance. Magn Reson Med 2011;66:505-519. doi: 10.1002/mrm.22999.

8. Mayer D, Levin YS, Hurd RE, Glover GH, Spielman DM. Fast metabolic imaging of systems with sparse spectra: Application for hyperpolarized13C imaging. Magn Reson Med 2006;56:932-937. doi: 10.1002/mrm.21025.

9. Levin YS, Mayer D, Yen Y-F, Hurd RE, Spielman DM. Optimization of fast spiral chemical shift imaging using least squares reconstruction: Application for hyperpolarized13C metabolic imaging. Magn Reson Med 2007;58:245-252. doi: 10.1002/mrm.21327.

10. Cunningham $\mathrm{CH}$, Chen $\mathrm{AP}$, Lustig $\mathrm{M}$, et al. Pulse sequence for dynamic volumetric imaging of hyperpolarized metabolic products. Journal of Magnetic Resonance 2008;193:139-146. doi: 10.1016/j.jmr.2008.03.012.

11. Schmidt R, Laustsen C, Dumez J-N, Kettunen MI, Serrao EM, Marco-Rius I, Brindle KM, Ardenkjaer-Larsen JH, Frydman L. Accepted Manuscript. Journal of Magnetic Resonance 2014:1-31. doi: 10.1016/j.jmr.2013.12.013.

12. Kurhanewicz J, Vigneron DB, Brindle K, et al. Analysis of cancer metabolism by imaging hyperpolarized nuclei: prospects for translation to clinical research. Neoplasia 2011;13:8197. doi: 10.1593/neo.101102. 
13. Tyler DJ. Cardiovascular Applications of Hyperpolarized MRI. Curr Cardiovasc Imaging Rep 2011;4:108-115. doi: 10.1007/s12410-011-9066-8.

14. Nelson SJ, Kurhanewicz J, Vigneron DB, et al. Metabolic imaging of patients with prostate cancer using hyperpolarized $\left[1-{ }^{13} \mathrm{C}\right]$ pyruvate. Sci Transl Med 2013;5:198ra108. doi: 10.1126/scitransImed. 3006070 .

15. Ball DR, Cruickshank R, Carr CA, Stuckey DJ, Lee P, Clarke K, Tyler DJ. Metabolic imaging of acute and chronic infarction in the perfused rat heart using hyperpolarised [113C]pyruvate. NMR in biomedicine 2013;26:1441-1450. doi: 10.1002/nbm.2972.

16. Comment A, van den Brandt B, Uffmann K, Kurdzesau F, Jannin S, Konter JA, Hautle $P$, Wenckebach WT, Gruetter R, van der Klink JJ. Design and performance of a DNP prepolarizer coupled to a rodent MRI scanner. Concepts in Magnetic Resonance Part B: Magnetic Resonance Engineering 2007;31:255-269.

17. Jannin S, Comment A, Kurdzesau F, Konter JA, Hautle $P$, van den Brandt $B$, van der Klink JJ. A $140 \mathrm{GHz}$ prepolarizer for dissolution dynamic nuclear polarization. The Journal of Chemical Physics 2008;128:241102. doi: 10.1063/1.2951994.

18. Jannin S, Bornet A, Melzi R, Bodenhausen G. High field dynamic nuclear polarization at 6.7 T: Carbon-13 polarization above $70 \%$ within 20 min. Chemical Physics Letters 2012;549:99-102. doi: 10.1016/j.cplett.2012.08.017.

19. Lumata LL, Martin R, Jindal AK, Kovacs Z, Conradi MS, Merritt ME. Development and performance of a 129-GHz dynamic nuclear polarizer in an ultra-wide bore superconducting magnet. MAGMA 2014. doi: 10.1007/s10334-014-0455-2.

20. Batel M, Krajewski M, Weiss K, et al. A multi-sample $94 \mathrm{GHz}$ dissolution dynamic-nuclearpolarization system. J. Magn. Reson. 2011;214:166-174. doi: 10.1016/j.jmr.2011.11.002.

21. Crémillieux Y, Goutailler F, Montcel B, Grand D, Vermeulen G, Wolf P-E. A Super-Wide Bore DNP System for Multiple Sample Polarization: Cryogenic Performance and Polarization at Low Temperature. Appl Magn Reson 2012;43:167-180. doi: 10.1007/s00723-012-0354-5.

22. Cheng T, Capozzi A, Takado Y, Balzan R, Comment A. Over $35 \%$ liquid-state $13 \mathrm{C}$ polarization obtained via dissolution dynamic nuclear polarization at $7 \mathrm{~T}$ and $1 \mathrm{~K}$ using ubiquitous nitroxyl radicals. Phys Chem Chem Phys 2013;15:20819-20822. doi: 10.1039/c3cp53022a.

23. Bornet A, Melzi R, Jannin S, Bodenhausen G. Cross Polarization for Dissolution Dynamic Nuclear Polarization Experiments at Readily Accessible Temperatures 1.2. Appl Magn Reson 2012;43:107-117. doi: 10.1007/s00723-012-0358-1.

24. Batel M, Krajewski M, Däpp A, Hunkeler A, Meier BH, Kozerke S, Ernst M. Dissolution dynamic nuclear polarization efficiency enhanced by Hartmannâ€"Hahn cross polarization. Chemical Physics Letters 2012;554:72-76. doi: 10.1016/j.cplett.2012.10.018.

25. Bornet A, Milani J, Vuichoud B, Linde AJP, Bodenhausen G, Jannin S. Microwave 
frequency modulation to enhance Dissolution Dynamic Nuclear Polarization. Chemical Physics Letters 2014;602:63-67. doi: 10.1016/j.cplett.2014.04.013.

26. Ardenkjaer-Larsen JH, Leach AM, Clarke N, Urbahn J, Anderson D, Skloss TW. Dynamic nuclear polarization polarizer for sterile use intent Rizi R, editor. NMR in biomedicine 2011;24:927-932. doi: 10.1002/nbm.1682.

27. Sigfridsson A, Weiss K, Wissmann L, Busch J, Krajewski M, Batel M, Batsios G, Ernst M, Kozerke $S$. Hybrid multiband excitation multiecho acquisition for hyperpolarized (13) C spectroscopic imaging. Magn Reson Med 2014;73:1713-1717. doi: 10.1002/mrm.25294.

28. Reeder SB, Wen ZF, Yu HZ, Pineda AR, Gold GE, MarkI M, Pelc NJ. Multicoil Dixon chemical species separation with an iterative least-squares estimation method. Magn Reson Med 2004;51:35-45. doi: 10.1002/mrm.10675.

29. Hu S, Larson PEZ, VanCriekinge M, et al. Rapid sequential injections of hyperpolarized [1${ }^{13} \mathrm{C}$ ]pyruvate in vivo using a sub-kelvin, multi-sample DNP polarizer. Magnetic resonance imaging 2013;31:490-496. doi: 10.1016/j.mri.2012.09.002.

30. Peterson ET, Gordon JW, Erickson MG, Fain SB, Rowland IJ. Dynamic Nuclear Polarization System Output Volume Reduction Using Inert Fluids. J Magn Reson Imaging 2011;33:10031008. doi: 10.1002/jmri.22498.

31. Cheng T, Mishkovsky M, Bastiaansen JAM, Ouari O, Hautle $P$, Tordo $P$, van den Brandt B, Comment A. Automated transfer and injection of hyperpolarized molecules with polarization measurement prior to in vivoNMR. NMR in biomedicine 2013;26:1582-1588. doi: 10.1002/nbm.2993.

32. Milani J, Vuichoud B, Bornet A, Mieville P, Mottier R, Jannin S, Bodenhausen G. A magnetic tunnel to shelter hyperpolarized fluids. Rev Sci Instrum 2015;86. doi:

10.1063/1.4908196. 


\section{Figure legends}

Figure 1. Schematic of DNP setup (a) including wide-bore magnet (1), helium dewar (2), vacuum pumps (3), microwave source (4), cryostat (5), DNP insert skeleton (6), sample collector (7), water heater (8), dissolution valve/connector (9), sample space (10), sample sticks (11). Insert containing one sample stick (b) and close-up (c) with waveguide (12), heat baffles (13), helium level sensor (14), NMR coil (15), and sample cup (16).

Figure 2. Details of sample stick configuration during polarization and dissolution. Stick tube (1), lifter in the upper position (2), inlet tube (3), outlet tube (4), dissolution coupling (5), sample cup during dissolution (6), liquid helium level (7) and sample cup during polarization (8).

Figure 3. Microwave activity, temperature, helium level* and NMR signal during polarization/dissolution of stick \#1-4 as a function of time ( $t=0$ refers to start of single-shot mode). Intermittent temperature increase upon dissolution is rapidly equilibrated. Helium consumption in single-shot mode was approximately $115 \mathrm{ml} / \mathrm{h}$. The NMR signal is corrected for differences in coil loading and sample volume (black curve). $\left({ }^{*}\right.$ the helium level sensor has a limited range and hence a decrease in helium level is only seen after $60 \mathrm{~min}$ as indicted by the dashed box).

Figure 4. Polarization and $\mathrm{T}_{1}$ time constants (mean $\pm \mathrm{SD}$ ) of [1-13C] pyruvate samples of sticks \#1-4 at t=30 sec after dissolution. Stick numbers refer to physical sticks. The order of dissolution and position during 5-7 runs per stick was randomized.

Figure 5. In-vivo imaging example. Maps of [1-13C] pyruvate, [1-13C] lactate and bicarbonate as well as signal intensity time curves acquired at $0,20,40$ and $60 \mathrm{~min}$ in the same animal demonstrate good reproducibility. 


\section{Figures}

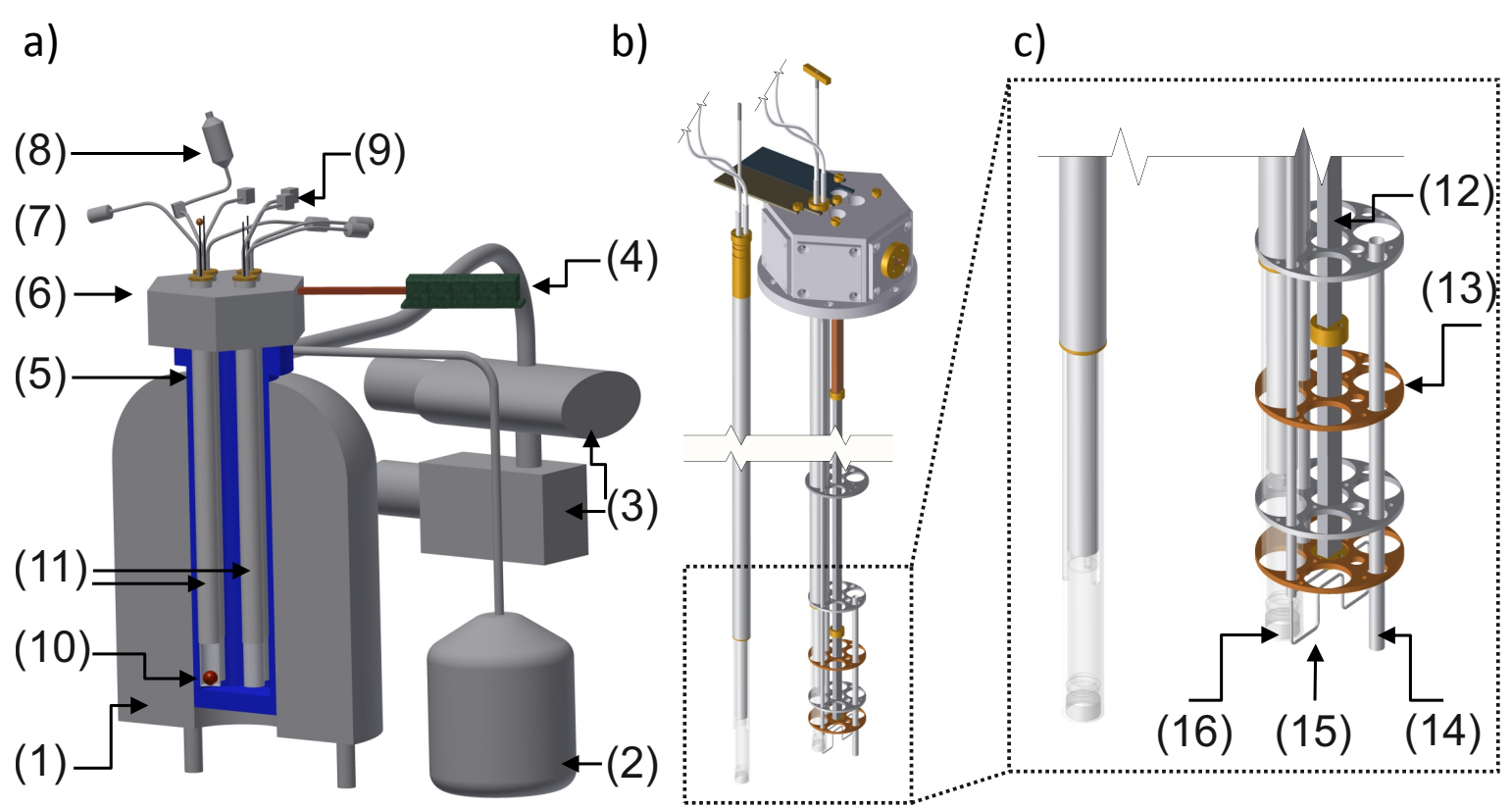

Figure 1. Schematic of DNP setup (a) including wide-bore magnet (1), helium dewar (2), vacuum pumps (3), microwave source (4), cryostat (5), DNP insert skeleton (6), sample collector (7), water heater (8), dissolution valve/connector (9), sample space (10), sample sticks (11). Insert containing one sample stick (b) and close-up (c) with waveguide (12), heat baffles (13), helium level sensor (14), NMR coil (15), and sample cup (16). 


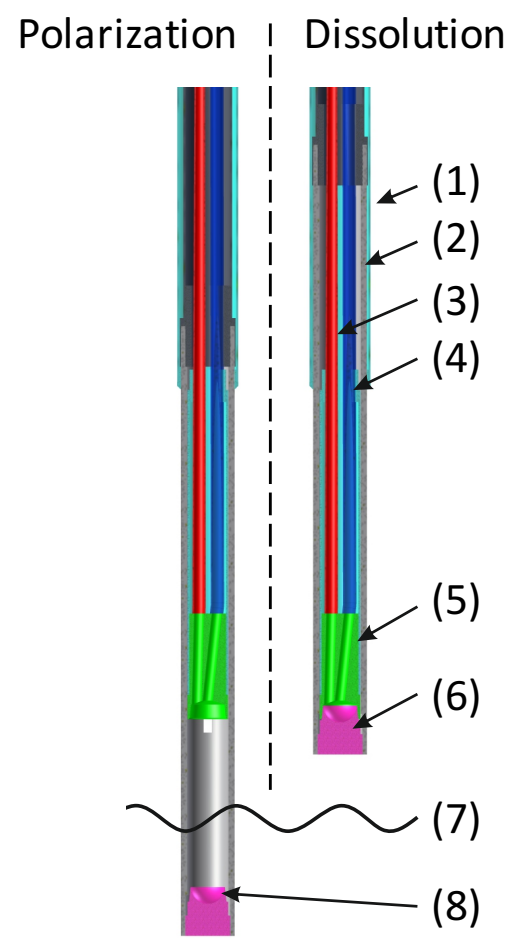

Figure 2. Details of sample stick configuration during polarization and dissolution. Stick tube (1), lifter in the upper position (2), inlet tube (3), outlet tube (4), dissolution coupling (5), sample cup during dissolution (6), liquid helium level (7) and sample cup during polarization (8). 


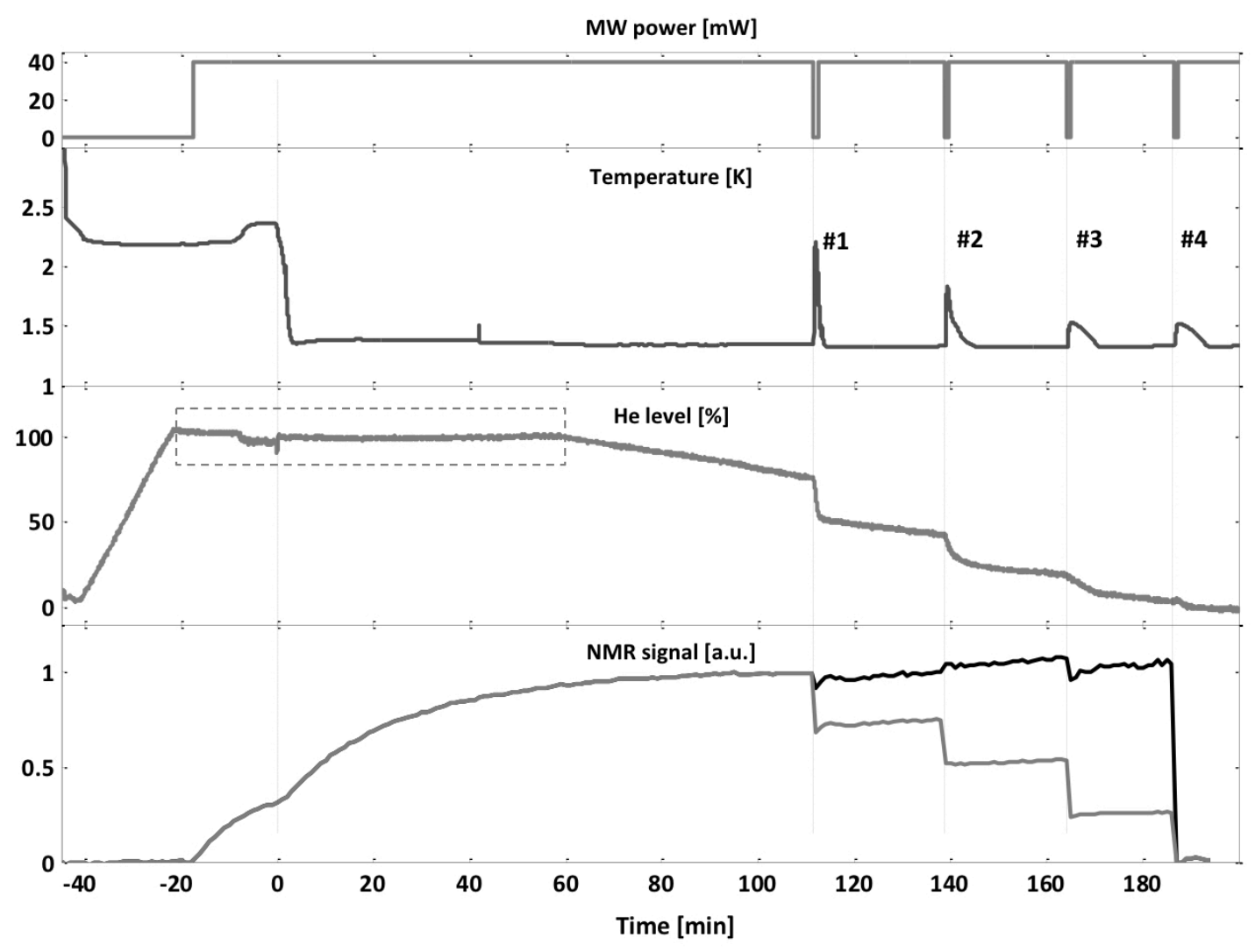

Figure 3. Microwave activity, temperature, helium level* and NMR signal during polarization/dissolution of stick \#1-4 as a function of time ( $\mathrm{t}=0$ refers to start of single-shot mode). Intermittent temperature increase upon dissolution is rapidly equilibrated. Helium consumption in single-shot mode was approximately $115 \mathrm{ml} / \mathrm{h}$. The NMR signal is corrected for differences in coil loading and sample volume (black curve). (* the helium level sensor has a limited range and hence a decrease in helium level is only seen after 60 min as indicted by the dashed box). 

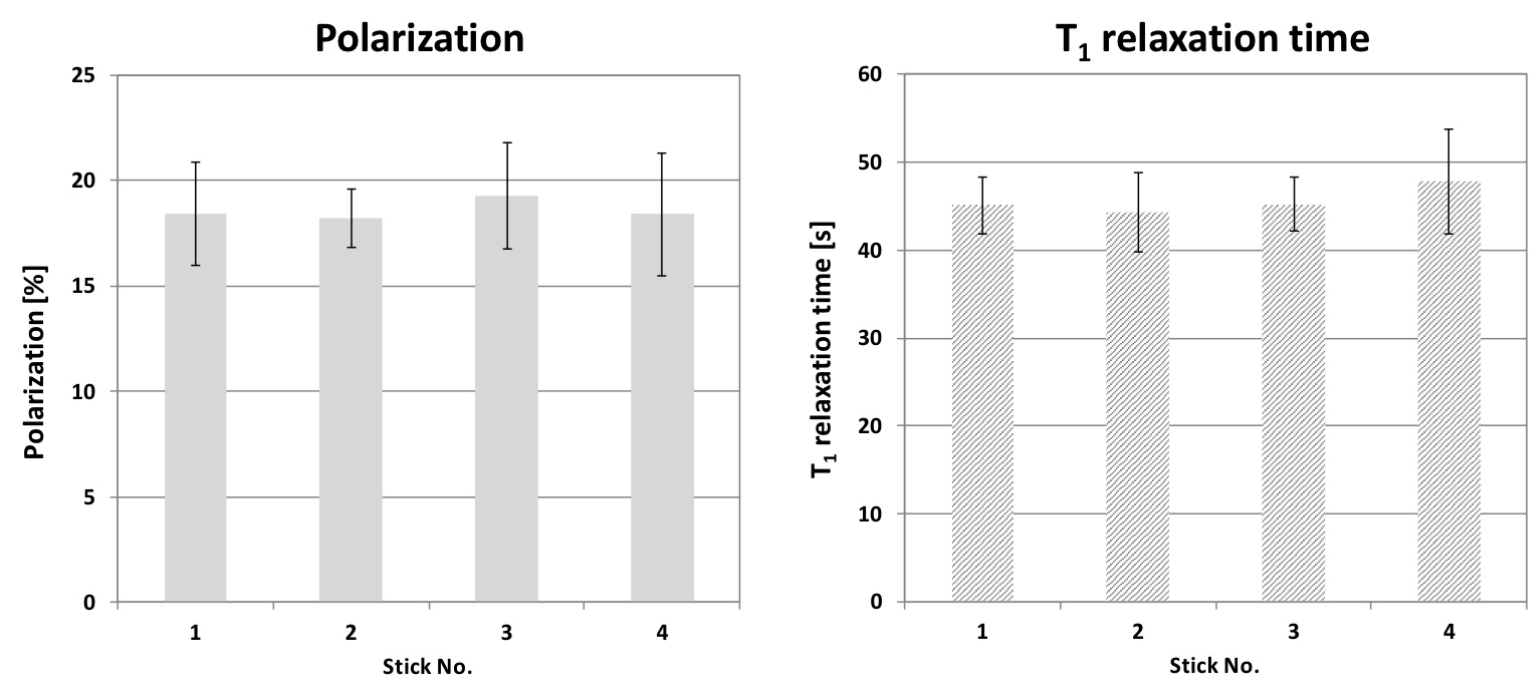

Figure 4. Polarization and $\mathrm{T}_{1}$ time constants (mean $\pm \mathrm{SD}$ ) of [1-13C] pyruvate samples of sticks \#1-4 at $\mathrm{t}=30 \mathrm{sec}$ after dissolution. Stick numbers refer to physical sticks. The order of dissolution and position during 5-7 runs per stick was randomized. 


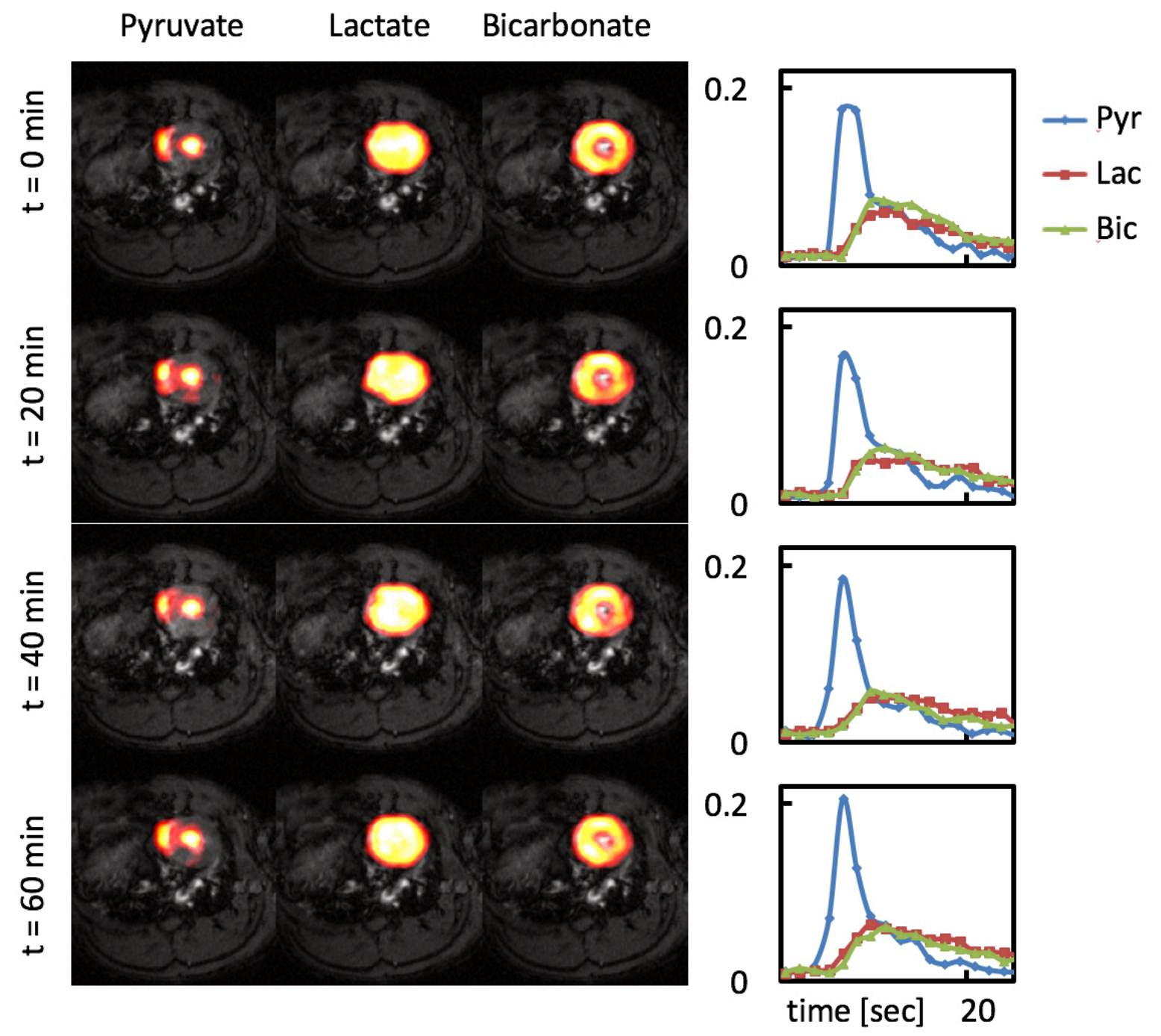

Figure 5. In-vivo imaging example. Maps of pyruvate, lactate and bicarbonate as well as signal intensity time curves acquired at 0,20, 40 and $60 \mathrm{~min}$ in the same animal demonstrate good reproducibility. 